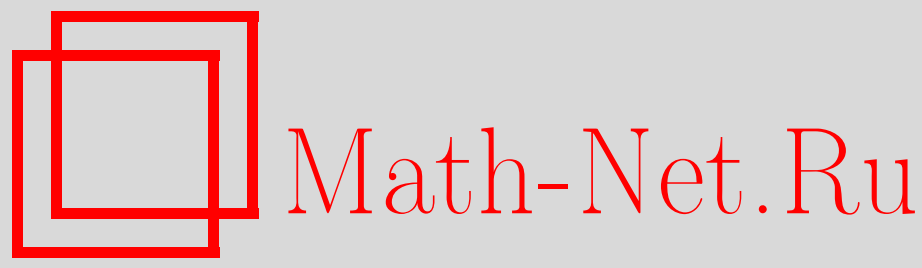

А. А. Элиович, В. И. Санюк, Некоторые аспекты применения полинорм в теории поля, ТМФ, 2010, том 162, номер 2, 163-178

DOI: https://doi.org/10.4213/tmf6462

Использование Общероссийского математического портала Math-Net.Ru подразумевает, что вы прочитали и согласны с пользовательским соглашением http://www. mathnet.ru/rus/agreement

Параметры загрузки:

IP : 54.80 .73 .141

26 апреля 2023 г., 14:38:49

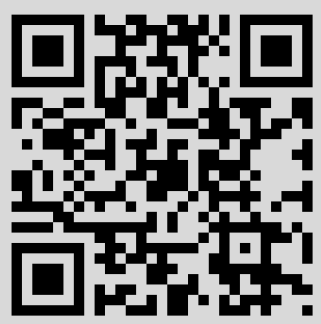




\title{
НЕКОТОРЫЕ АСПЕКТЫ ПРИМЕНЕНИЯ ПОЛИНОРМ В ТЕОРИИ ПОЛЯ
}

\begin{abstract}
Рассматриваются некоторые возможности применения в физике псевдонорм степени выше 2 (полинорм) на гиперкомплексных алгебрах, прежде всего на примере алгебры бикватернионов. При этом на ряд известных вопросов можно посмотреть под новым углом зрения. В частности, показано, что рассмотрение 4-нормы в теории поля делает естественным переход от электродинамики Максвелла к нелинейной электродинамике Борна-Инфельда. Кроме того, алгебраический подход показывает естественность добавления предложенного Скирмом нелинейного члена в мезонный лагранжиан ядерных сил. При этом выявляется целесообразность введения единственного дополнительного члена четвертого порядка в лагранжиан Скирма, что может улучшить свойства модели.
\end{abstract}

Ключевые слова: гиперкомплексная алгебра, бикватернион, мультипликативная полинорма, нелинейная теория поля, электродинамика Борна-Инфельда, модель Скирма.

\section{1. ВВЕДЕНИЕ}

В последнее время растет интерес к изучению связей между гиперкомплексными алгебрами, геометрией и физикой. Примеров применения гиперкомплексных алгебр в физике не так мало [1], [2]. Кватернионы - естественный язык для описания вращений твердого тела и для ньютоновой механики в целом, поскольку группа их автоморфизмов $S U(2)$ локально изоморфна группе трехмерных вращений $S O(3)$. Это построение, так называемая теория винтов, было создано Клиффордом, Котельниковым и Штуди [3]-[6]. Сходным образом язык бикватернионов (комплексных кватернионов) достаточно органичен для теории относительности, поскольку группа их автоморфизмов $S L(2, \mathbb{C})$ локально изоморфна группе Лоренца $S O(1,3)$. Как следствие, уравнения, выражающие условие аналитичности бикватернионных переменных (уравнения Фютера), совпадают с уравнениями Максвелла (см. [1], [7]). С другой стороны, матрицы Паули образуют алгебру, изоморфную бикватернионам, что показывает связь последней алгебры с квантовой механикой, в частности с теорией спиноров. Есть и ряд других примеров.

*Российский университет дружбы народов, Москва, Россия.

E-mail: eliovich@mail.ru,vsanyuk@mail.ru 
Однако применение гиперкомплексных алгебр в физике не носит систематического характера. Одновременно во всех известных успешных результатах никак не используется тот факт, что алгебра бикватернионов наряду с комплексной псевдонормой второго порядка обладает и вещественной псевдонормой четвертого порядка. Возникает вопрос, что может дать учет этого факта, т. е. введение в рассмотрение полинорм сверх обычных квадратичных норм.

Статья организована следующим образом. В разделе 2 вводятся основные математические конструкции на ассоциативных алгебрах на примере алгебры бикватернионов. В разделе 3 демонстрируются некоторые возможности применения полученного аппарата в физике, прежде всего в нелинейной теории поля.

\section{2. АЛГЕБРЫ И ПОЛИНОРМЫ}

2.1. Кватернионы и бикватернионы: краткая сводка. Кватернионы $H$ (см., например, [8]) - это ближайшее обобщение комплексных чисел. Они были придуманы Гамильтоном в 1843 г. для описания вращений трехмерного пространства (подобно тому, как комплексные числа могут служить для описания преобразований плоскости). Кватернионы суть гиперкомплексные числа размерности 4:

$$
\mathbf{a}=a_{0}+a_{1} \mathbf{q}_{1}+a_{2} \mathbf{q}_{2}+a_{3} \mathbf{q}_{3},
$$

где $a_{i}$ - вещественные числа, а орты $\mathbf{q}_{i}$ перемножаются согласно правилу умножения

$$
\mathbf{1} \mathbf{q}_{k}=\mathbf{q}_{k} \mathbf{1}=\mathbf{q}_{k}, \quad \mathbf{q}_{j} \mathbf{q}_{k}=-\delta_{j k} \mathbf{1}+\varepsilon_{j k n} \mathbf{q}_{n},
$$

где $\delta_{j k}$ и $\varepsilon_{j k n}$ - символы Кронекера и Леви-Чивиты, $j, k, n=1,2,3$. Умножение кватернионов некоммутативно, но ассоциативно (т. е. не зависит от расстановки скобок).

Кроме умножения и сложения, на кватернионах, как и на комплексных числах, определена важная операция сопряжения

$$
\overline{\mathbf{a}}=a_{0}-a_{1} \mathbf{q}_{1}-a_{2} \mathbf{q}_{2}-a_{3} \mathbf{q}_{3} .
$$

Кватернионное сопряжение является инволюцией, линейно и переставляет порядок сомножителей, т. е. является антиавтоморфизмом:

$$
\overline{\overline{\mathbf{a}}}=\mathbf{a}, \quad \overline{\lambda \mathbf{a}+\mu \mathbf{b}}=\lambda \overline{\mathbf{a}}+\mu \overline{\mathbf{b}}, \quad \overline{\mathbf{a b}}=\overline{\mathbf{b}} \overline{\mathbf{a}},
$$

где $\lambda, \mu$ - вещественные числа. Благодаря этим свойствам следующие выражения не меняются при сопряжении:

$$
\begin{gathered}
\operatorname{Re} \mathbf{a}=\frac{1}{2}(\mathbf{a}+\overline{\mathbf{a}}) \quad(\text { реальная часть }), \\
N_{\mathrm{r}}(\mathbf{a})=\mathbf{a} \cdot \overline{\mathbf{a}}, \quad N_{\mathrm{l}}(\mathbf{a}) \equiv N_{\mathrm{r}}(\overline{\mathbf{a}})=\overline{\mathbf{a}} \cdot \mathbf{a} \quad(\text { правая и левая 2-нормы). }
\end{gathered}
$$

При этом Re $\overline{\mathbf{a}} \equiv \operatorname{Re} \mathbf{a}$, а левая и правая нормы совпадают (это свойство выполняется не во всех алгебрах):

$$
N_{\mathrm{r}}(\mathbf{a})=N_{\mathrm{l}}(\mathbf{a}) \equiv N(\mathbf{a})=a_{0}^{2}+a_{1}^{2}+a_{2}^{2}+a_{3}^{2} .
$$


Отсюда видно, что норма кватернионов положительно определена, а их метрика имеет сигнатуру +4. Самое замечательное свойство кватернионов - мультипликативность их нормы, т. е. норма произведения кватернионов равна произведению их норм:

$$
N(\mathbf{a b})=N(\mathbf{a}) N(\mathbf{b})
$$

Этот факт лежит в основе геометрических интерпретаций кватернионов. Кроме того, благодаря этому свойству для вещественных чисел имеет место замечательное тождество четырех квадратов: правильно записанное произведение сумм четырех квадратов есть снова сумма четырех квадратов.

Уникальность кватернионов вытекает из классических теорем об исключительных алгебрах (см. [8]-[10]). Высшая из исключительных алгебр - алгебра октав размерности 8 - также обладает положительно-определенной (сигнатура метрики +8 ) и мультипликативной нормой. Однако алгебра октав уже не ассоциативна, что затрудняет ее применение в геометрии и физике. Сохраняется лишь ослабленная ассоциативность (альтернативность): в октавах любые два элемента порождают ассоциативную подалгебру. Октавы связаны с исключительными группами Ли, значение которых для физики в последнее время растет, и с наиболее глубокими областями проективной геометрии [2], [11]. В целом роль октав в физике остается не вполне проясненной. Октавы и свойство альтернативности затронуты в данной статье лишь для прояснения общей теории и особенностей кватернионов и бикватернионов.

Бикватернионы $B$ суть кватернионы, определенные над полем комплексных чисел $\left(\mathbf{i}_{0}^{2}=-1\right)$. Следовательно, эти числа можно по-прежнему записывать в виде (1), но коэффициенты $a_{i}$ при этом - уже комплексные числа. Однако в настоящей работе принята более свободная трактовка бикватернионов как 8-мерной алгебры над полем вещественных чисел, состоящей из двух блоков:

$$
\mathbf{a}=a+k \cdot \mathbf{i}_{0}
$$

или, в развернутой записи,

$$
\mathbf{a}=a_{0}+a_{1} \mathbf{q}_{1}+a_{2} \mathbf{q}_{2}+a_{3} \mathbf{q}_{3}+k_{0} \mathbf{i}_{0}+k_{1} \mathbf{i}_{1}+k_{2} \mathbf{i}_{2}+k_{3} \mathbf{i}_{3},
$$

где $a, k$ - кватернионы с вещественными коэффициентами. Величина $\mathbf{i}_{0}$ является внешней единицей, коммутирующей с кватернионами $a, k, \mathbf{i}_{0}^{2}=-1$; единицы $\mathbf{i}_{j}-$ результат внешнего (тензорного) произведения $\mathbf{i}_{0} \otimes \mathbf{q}_{j}$. Отсюда вытекает правило умножения бикватернионов в блочном и табличном виде (см. таблицу, где левый верхний угол заодно представляет таблицу умножения кватернионов):

$$
\left(a+k \cdot \mathbf{i}_{0}\right)\left(b+l \cdot \mathbf{i}_{0}\right)=a b-k l+(a l+k b) \cdot \mathbf{i}_{0} .
$$

2.2. 2-Норма и система сопряжений для бикватернионов. Ключом к пониманию свойств гиперкомплексной алгебры является не столько таблица умножения, сколько система заданных на ней сопряжений. Учитывая, что бикватернионы 


\begin{tabular}{|c|cccc|cccc|}
\hline$\times$ & 1 & $\mathbf{q}_{1}$ & $\mathbf{q}_{2}$ & $\mathbf{q}_{3}$ & $\mathbf{i}_{0}$ & $\mathbf{i}_{1}$ & $\mathbf{i}_{2}$ & $\mathbf{i}_{3}$ \\
\hline 1 & 1 & $\mathbf{q}_{1}$ & $\mathbf{q}_{2}$ & $\mathbf{q}_{3}$ & $\mathbf{i}_{0}$ & $\mathbf{i}_{1}$ & $\mathbf{i}_{2}$ & $\mathbf{i}_{3}$ \\
$\mathbf{q}_{1}$ & $\mathbf{q}_{1}$ & -1 & $\mathbf{q}_{3}$ & $-\mathbf{q}_{2}$ & $\mathbf{i}_{1}$ & $-\mathbf{i}_{0}$ & $\mathbf{i}_{3}$ & $-\mathbf{i}_{2}$ \\
$\mathbf{q}_{2}$ & $\mathbf{q}_{2}$ & $-\mathbf{q}_{3}$ & -1 & $\mathbf{q}_{1}$ & $\mathbf{i}_{2}$ & $-\mathbf{i}_{3}$ & $-\mathbf{i}_{0}$ & $\mathbf{i}_{1}$ \\
$\mathbf{q}_{3}$ & $\mathbf{q}_{3}$ & $\mathbf{q}_{2}$ & $-\mathbf{q}_{1}$ & -1 & $\mathbf{i}_{3}$ & $\mathbf{i}_{2}$ & $-\mathbf{i}_{1}$ & $-\mathbf{i}_{0}$ \\
\hline $\mathbf{i}_{0}$ & $\mathbf{i}_{0}$ & $\mathbf{i}_{1}$ & $\mathbf{i}_{2}$ & $\mathbf{i}_{3}$ & -1 & $-\mathbf{q}_{1}$ & $-\mathbf{q}_{2}$ & $-\mathbf{q}_{3}$ \\
$\mathbf{i}_{1}$ & $\mathbf{i}_{1}$ & $-\mathbf{i}_{0}$ & $\mathbf{i}_{3}$ & $-\mathbf{i}_{2}$ & $-\mathbf{q}_{1}$ & 1 & $-\mathbf{q}_{3}$ & $\mathbf{q}_{2}$ \\
$\mathbf{i}_{2}$ & $\mathbf{i}_{2}$ & $-\mathbf{i}_{3}$ & $-\mathbf{i}_{0}$ & $\mathbf{i}_{1}$ & $-\mathbf{q}_{2}$ & $\mathbf{q}_{3}$ & 1 & $-\mathbf{q}_{1}$ \\
$\mathbf{i}_{3}$ & $\mathbf{i}_{3}$ & $\mathbf{i}_{2}$ & $-\mathbf{i}_{1}$ & $-\mathbf{i}_{0}$ & $-\mathbf{q}_{3}$ & $-\mathbf{q}_{2}$ & $\mathbf{q}_{1}$ & 1 \\
\hline
\end{tabular}

представляют собой прямо удвоенные кватернионы, аналогичное кватернионное сопряжение $\overline{\mathbf{a}}$ для них можно ввести по очевидному правилу:

$$
\begin{aligned}
& \overline{\mathbf{a}}=\alpha_{0}-\alpha_{1} \mathbf{q}_{1}-\alpha_{2} \mathbf{q}_{2}-\alpha_{3} \mathbf{q}_{3}, \\
& \overline{\mathbf{a}}=a_{0}-a_{1} \mathbf{q}_{1}-a_{2} \mathbf{q}_{2}-a_{3} \mathbf{q}_{3}+k_{0} \mathbf{i}_{0}-k_{1} \mathbf{i}_{1}-k_{2} \mathbf{i}_{2}-k_{3} \mathbf{i}_{3}, \\
& \overline{\mathbf{a}}=\overline{a+k \cdot \mathbf{i}_{0}}=\bar{a}+\bar{k} \cdot \mathbf{i}_{0},
\end{aligned}
$$

где первое выражение представляет собой запись с комплексными коэффициентами, второе - полную запись с вещественными коэффициентами, а третье - сокращенную запись.

С помощью данного сопряжения на алгебре бикватернионов можно определить квадратичную 2-норму, которая теперь будет не вещественным, а комплексным числом:

$$
N_{2}(\mathbf{a})=\mathbf{a} \cdot \overline{\mathbf{a}}=\overline{\mathbf{a}} \cdot \mathbf{a} .
$$

В покомпонентном виде 2-норма бикватернионов имеет вид

$$
N_{2}(\mathbf{a})=\left(a_{0}^{2}+a_{1}^{2}+a_{2}^{2}+a_{3}^{2}-k_{0}^{2}-k_{1}^{2}-k_{2}^{2}-k_{3}^{2}\right)+4 \mathbf{i}_{0}\left(a_{0} k_{0}+a_{1} k_{1}+a_{2} k_{2}+a_{3} k_{3}\right) .
$$

Комплексная 2-норма в алгебре бикватернионов, как и ее аналог в алгебре биоктав (комплексных октав), также обладает замечательным свойством мультипликативности (7). На ее основе можно ввести и вещественную псевдонорму четвертого порядка, которая наследует свойство мультипликативности (см. ниже, а также работы [12], [13]).

Для построения вещественной 4-нормы и 4-скалярного произведения одного базового кватернионного сопряжения недостаточно. Поэтому введем второе, дуалъное кватернионное сопряжение (записи в той же последовательности, что и в (11)):

$$
\begin{aligned}
& \tilde{\mathbf{a}}=\alpha_{0}^{*}-\alpha_{1}^{*} \mathbf{q}_{1}-\alpha_{2}^{*} \mathbf{q}_{2}-\alpha_{3}^{*} \mathbf{q}_{3}, \\
& \tilde{\mathbf{a}}=a_{0}-a_{1} \mathbf{q}_{1}-a_{2} \mathbf{q}_{2}-a_{3} \mathbf{q}_{3}-k_{0} \mathbf{i}_{0}+k_{1} \mathbf{i}_{1}+k_{2} \mathbf{i}_{2}+k_{3} \mathbf{i}_{3}, \\
& \tilde{\mathbf{a}}=a \widetilde{+k \cdot \mathbf{i}_{0}}=\bar{a}-\bar{k} \cdot \mathbf{i}_{0} .
\end{aligned}
$$

Преобразование а̃ (14) также может считаться сопряжением, поскольку, как и $\mathbf{a}(11)$, является инволюционным антиавтоморфизмом. Однако, в отличие от базового сопряжения, вообще говоря, аã $\neq$ ãa. 
Теперь рассмотрим комбинацию сопряжений $\overline{\tilde{a}}$ :

$$
\begin{aligned}
& \overline{\tilde{\mathbf{a}}}=a_{0}^{*}+a_{1}^{*} \mathbf{q}_{1}+a_{2}^{*} \mathbf{q}_{2}+a_{3}^{*} \mathbf{q}_{3}, \\
& \overline{\tilde{\mathbf{a}}}=a_{0}+a_{1} \mathbf{q}_{1}+a_{2} \mathbf{q}_{2}+a_{3} \mathbf{q}_{3}-k_{0} \mathbf{i}_{0}-k_{1} \mathbf{i}_{1}-k_{2} \mathbf{i}_{2}-k_{3} \mathbf{i}_{3}, \\
& \overline{\tilde{\mathbf{a}}}=\overline{a+k \cdot \mathbf{i}_{0}}=a-k \cdot \mathbf{i}_{0} .
\end{aligned}
$$

Отсюда видно, что преобразование а̃ есть комплексное сопряжение, внешнее для кватернионов $a, k$. Обозначим его $\mathbf{a}^{*}$. Оно является инволюцией и автоморфизмом (а не антиавтоморфизмом):

$$
\left(\mathbf{a}^{*}\right)^{*}=\mathbf{a}, \quad(\mathbf{a b})^{*}=\mathbf{a}^{*} \mathbf{b}^{*} .
$$

Важное преимущество базового сопряжения $\overline{\mathbf{a}}$ (11) перед дуальным сопряжением а̃ в том, что гиперкомплексные числа, инвариантные относительно основного сопряжения $\overline{\mathbf{a}}$ (их можно назвать реальными относительно него), содержат в себе только орты 1 и $\mathbf{i}_{\mathbf{0}}$ и поэтому, подобно вещественным числам, коммутируют с любыми числами алгебры. Данное свойство базового кватернионного сопряжения а̄ и является источником ряда хороших свойств алгебр кватернионов и бикватернионов. Лишь немногие алгебры располагают таким "хорошим" сопряжением, которое называют скалярным или централъным. Алгебры с центральным сопряжением и их основные свойства изучены в работах [12], [13].

2.3. 2-Скалярное и 2-векторное произведения в алгебрах $H$ и $B$. Ниже нам потребуются не только 2-норма (12), (13), но и другие квадратичные конструкции для алгебр кватернионов $H$ и бикватернионов $B$. Введем 2-скалярное произведение (для бикватернионов оно является не вещественным, а комплексным числом):

$$
N(\mathbf{a}+\mathbf{b})=N(\mathbf{a})+N(\mathbf{b})+2(\mathbf{a}, \mathbf{b})
$$

и, следовательно,

$$
(\mathbf{a}, \mathbf{b})=\frac{1}{2}(N(\mathbf{a}+\mathbf{b})-N(\mathbf{a})-N(\mathbf{b}))
$$

Очевидно,

$$
(\mathbf{a}, \mathbf{b})=(\mathbf{b}, \mathbf{a}), \quad(\mathbf{a}, \mathbf{a})=N(\mathbf{a}) .
$$

Легко выразить 2-скалярное произведение напрямую через операции алгебры:

$$
(\mathbf{a}, \mathbf{b})=\operatorname{Re}(\mathbf{a} \overline{\mathbf{b}})=\frac{1}{2}(\mathbf{a} \overline{\mathbf{b}}+\mathbf{b} \overline{\mathbf{a}})=\operatorname{Re}(\overline{\mathbf{a}} \mathbf{b})=\frac{1}{2}(\overline{\mathbf{a}} \mathbf{b}+\overline{\mathbf{b}} \mathbf{a})=(\overline{\mathbf{a}}, \overline{\mathbf{b}}) .
$$

Таким образом, в силу свойств 2-нормы на алгебрах $H$ и $B$ левое и правое скалярные произведения совпадают.

Введем теперь левое (l) и правое (r) векторные произведения; это уже не числа, а элементы алгебры кватернионов или бикватернионов:

$$
\begin{aligned}
\langle\mathbf{a}, \mathbf{b}\rangle_{1} & \equiv \operatorname{Im}(\overline{\mathbf{a}} \mathbf{b})=\frac{1}{2}(\overline{\mathbf{a}} \mathbf{b}-\overline{\mathbf{b}} \mathbf{a}), \\
\langle\mathbf{a}, \mathbf{b}\rangle_{\mathrm{r}} & \equiv \operatorname{Im}(\mathbf{a} \overline{\mathbf{b}})=\frac{1}{2}(\mathbf{a} \overline{\mathbf{b}}-\mathbf{b} \overline{\mathbf{a}}) .
\end{aligned}
$$


С очевидностью,

$$
\langle\mathbf{b}, \mathbf{a}\rangle=-\langle\mathbf{a}, \mathbf{b}\rangle, \quad\langle\mathbf{a}, \mathbf{a}\rangle=0, \quad\langle\mathbf{a}, \mathbf{b}\rangle_{\mathrm{r}}=\langle\overline{\mathbf{a}}, \overline{\mathbf{b}}\rangle_{1}
$$

Отметим, что для векторных произведений

$$
\langle\mathbf{a}, \mathbf{b}\rangle \neq\langle\overline{\mathbf{a}}, \overline{\mathbf{b}}\rangle, \quad\langle\mathbf{a}, \mathbf{b}\rangle_{\mathrm{r}} \neq\langle\mathbf{a}, \mathbf{b}\rangle_{1}
$$

Однако, как показано в работе [12], в алгебрах $H$ и $B$ выполняется равенство

$$
\langle\mathbf{a}, \mathbf{b}\rangle^{2}=\langle\overline{\mathbf{a}}, \overline{\mathbf{b}}\rangle^{2}, \quad \text { т. е. } \quad\langle\mathbf{a}, \mathbf{b}\rangle_{\mathrm{r}}^{2}=\langle\mathbf{a}, \mathbf{b}\rangle_{1}^{2}
$$

В алгебрах кватернионов и бикватернионов верно тождество

$$
N_{2}(\mathbf{a}) N_{2}(\mathbf{b})=(\mathbf{a}, \mathbf{b})^{2}-\langle\mathbf{a}, \mathbf{b}\rangle^{2} .
$$

Далее, если не указан индекс r или l, мы будем иметь в виду правое векторное произведение.

2.4. Мультипликативные полинормы. В основе теории полинорм лежит идея распространить на полинормы условие мультипликативности 2-нормы $N(a \cdot b)=N(a) N(b)$. Это условие естественным образом связывает псевдонорму (форму) с умножением алгебры. В 1950-60-х годах вопрос о мультипликативности форм степени выше 2 поставил и решил Шафер [14], [15] (с дополнениями МакКриммона [16]), показав, что невырожденные мультипликативные псевдонормы можно построить только над альтернативными (в частности, ассоциативными) алгебрами.

Пусть $V$ - векторное пространство, возможно бесконечномерное, над полем $F$ характеристики 0 или $p>n$. Отображение $\mathbf{u} \rightarrow N(\mathbf{u}) V$ на $F$ называется формой cmепени $n$ на $V$ в случае

$$
N(\lambda \mathbf{u})=\lambda^{n} N(\mathbf{u}) \quad \forall \lambda \in F, \mathbf{u} \in V .
$$

ТеОРема ШАфеРА. Пусть $\mathbb{U}$ - алгебра с единицей, вообще говоря бесконечномерная, над полем F характеристики 0 или $p>n$. Для того чтобы на $\mathbb{U}$ существовала невырожденная форма $N$ степени $n>0$, допускающая композичию, необходимо и достаточно, чтобъ $\mathbb{U}$ являлась конечномерной сепарабельной альтернативной алгеброй, $\mathbb{U}=\mathbb{U}_{1} \oplus \cdots \oplus \mathbb{U}_{r}, \mathbb{U}_{i}-$ простая алгебра степени $m_{i}, i=1, \ldots, r$, где равенство $n=m_{1} f_{1}+\cdots+m_{r} f_{r}$ удовлетворяется при некоторых положительных иелых числах $f_{1}, \ldots, f_{r}$. Более того, форма $N$ на $\mathbb{U}$ задается соотношением

$$
N(\mathbf{u})=\left[n_{1}\left(u_{1}\right)\right]^{f_{1}} \cdots\left[n_{r}\left(u_{r}\right)\right]^{f_{r}},
$$

где $n_{j}\left(u_{j}\right)$ - форма, заданная на простой алгебре $\mathbb{U}_{j}$.

Здесь существенным является понятие невырожденности формы степени $n$, которое вводится следующим образом. 
1. С каждой $n$-нормой связывается $n$-линейная форма $n$-скалярного произведения от $n$ гиперкомплексных чисел по формуле Шафера

$$
\begin{aligned}
& \left(\mathbf{u}_{1}, \mathbf{u}_{2}, \ldots, \mathbf{u}_{n}\right)=\frac{1}{n !}\left[N\left(\mathbf{u}_{1}+\cdots+\mathbf{u}_{n}\right)-\sum_{i=1}^{n} N\left(\mathbf{u}_{1}+\cdots+\breve{\mathbf{u}}_{i}+\cdots+\mathbf{u}_{n}\right)+\right. \\
& \left.\quad+\sum_{i<j} N\left(\mathbf{u}_{1}+\cdots+\breve{\mathbf{u}}_{i}+\cdots+\breve{\mathbf{u}}_{j}+\cdots+\mathbf{u}_{n}\right)-\cdots+(-1)^{n-1} \sum_{i=1}^{n} N\left(\mathbf{u}_{i}\right)\right]
\end{aligned}
$$

где запись $\breve{\mathbf{u}}_{i}$ означает, что $\mathbf{u}_{i}$ опущен. Легко видеть, что

$$
N_{n}(\mathbf{u})=(\mathbf{u}, \mathbf{u}, \ldots, \mathbf{u})
$$

поскольку

$$
\sum_{k=0}^{n-1}(-1)^{k} C_{n}^{k}(n-k)^{n}=n ! .
$$

Форма $\left(\mathbf{u}_{1}, \mathbf{u}_{2}, \ldots, \mathbf{u}_{n}\right)$ имеет все свойства, которые естественно ожидать от полилинейного обобщения понятия скалярного произведения. Она вещественна, симметрична относительно любых перестановок векторов, линейна по каждому из них (в частности, обращается в нуль, если один из векторов равен нулю). Более правильно называть эту форму $n$-псевдоскалярным произведением, так как она в общем случае не является положительно определенной.

2. По определению Шафера формы степени $n$ называются невырожденными, если из условия, что $\left(\mathbf{u}_{1}, \mathbf{u}_{2}, \ldots, \mathbf{u}_{n}\right)=0$ для всех $\mathbf{u}_{2}, \ldots, \mathbf{u}_{n} \in V$, вытекает $\mathbf{u}_{1}=0$.

Итак, условие мультипликативности полинорм (вместе с условием их невырожденности) вносит существенное ограничение, значительно сужая круг рассматриваемых алгебр. Это ограничение довольно конструктивно с точки зрения применения алгебр в геометрии и физике, поскольку переход к алгебрам с псевдонормой (формой) выше квадратичной открывает слишком много степеней свободы.

2.5. 4-Норма и 4-скалярное произведение для бикватернионов. Мультипликативные вещественные 4-нормы в алгебрах бикватернионов и биоктав могут также быть построены на основе мультипликативных комплексных норм второго порядка [12]. В самом деле, умножив комплексную 2-норму этих алгебр на сопряженное комплексное число, мы получим вещественное число с наследуемым свойством мультипликативности:

$$
N_{4}(\mathbf{a})=N_{2}(\mathbf{a}) N_{2}(\mathbf{a})^{*}=\mathbf{a} \overline{\mathbf{a}}(\mathbf{a} \overline{\mathbf{a}})^{*}=\mathbf{a} \overline{\mathbf{a}} \mathbf{a}^{*} \overline{\mathbf{a}}^{*}=N_{2}(\mathbf{a}) N_{2}\left(\mathbf{a}^{*}\right) .
$$

Здесь * - комплексное, или двойное сопряжение $(\mathbf{a b})^{*}=\mathbf{a}^{*} \mathbf{b}^{*}($ см. (15)).

В сокращенном (блочном) виде 4-норма для алгебр бикватернионов равна

$$
N_{4}(\mathbf{a})=N_{4}\left(a+k \cdot \mathbf{i}_{0}\right)=\left(N_{2}(a)-N_{2}(k)\right)^{2}+4(a, k)^{2} .
$$

В покомпонентном виде 4-норма бикватернионов принимает вид

$$
N_{4}(\mathbf{a})=\left(a_{0}^{2}+a_{1}^{2}+a_{2}^{2}+a_{3}^{2}-k_{0}^{2}-k_{1}^{2}-k_{2}^{2}-k_{3}^{2}\right)^{2}+4\left(a_{0} k_{0}+a_{1} k_{1}+a_{2} k_{2}+a_{3} k_{3}\right)^{2} .
$$


Очевидно, что 4-норма неотрицательна. Но она не является положительно определенной (и не может являться в силу теоремы Фробениуса): из факта $N_{4}(\mathbf{a})=0$ не следует $\mathbf{a}=0$. Именно поэтому более корректно говорить о псевдонормах. Как легко видеть, чтобы 4-норма обращалась в нуль, нужно взять $k$ равным по 2-норме $a$ и при этом перпендикулярным ему: $(a, k)=0$. Например, $a=3 \mathbf{i}_{1}-2 \mathbf{i}_{3}, k=2 \mathbf{i}_{0}+3 \mathbf{i}_{2}$.

Формула, выражающая мультипликативность 4-нормы алгебры бикватернионов, в кватернионной записи выглядит так (с помощью (17) и ряда других кватернионных тождеств ее можно доказать напрямую):

$$
\begin{gathered}
\left(\left(N_{2}(a)-N_{2}(k)\right)^{2}+4(a, k)^{2}\right)\left(\left(N_{2}(b)-N_{2}(l)\right)^{2}+4(b, l)^{2}\right)= \\
\quad=\left(N_{2}(a b-k l)-N_{2}(a l+k b)\right)^{2}+4(a b-k l, a l+k b)^{2} .
\end{gathered}
$$

Это тождество можно записать и проверить в вещественных числах [12]. Подобно формулам суммы квадратов, это громоздкое тождество, случайное для вещественных чисел, отражает существование алгебры бикватернионов.

Для бикватернионов 4-норма, как можно показать [12], может быть представлена в ином виде, использующем дуальное сопряжение (14):

$$
\begin{gathered}
N_{4}(\mathbf{a})=\mathbf{a} \tilde{\mathbf{a}}(\mathbf{a} \tilde{\mathbf{a}})^{*}, \\
N_{4}(\mathbf{a})=N_{4}\left(a+k \cdot \mathbf{i}_{0}\right)=\left(N_{2}(a)+N_{2}(k)\right)^{2}+4\langle k, a\rangle^{2}, \\
\left(N_{2}(a)-N_{2}(k)\right)^{2}+4(a, k)^{2}=\left(N_{2}(a)+N_{2}(k)\right)^{2}+4\langle k, a\rangle^{2} .
\end{gathered}
$$

Зная 4-норму, можно ввести обратный элемент для алгебры бикватернионов. В самом деле, поскольку

$$
N_{4}(\mathbf{a})=N_{2}(\mathbf{a}) N_{2}^{*}(\mathbf{a})=\mathbf{a} \overline{\mathbf{a}} N_{2}^{*}(\mathbf{a}), \quad N_{4}(\overline{\mathbf{a}})=\overline{\mathbf{a}} \mathbf{a} N_{2}^{*}(\overline{\mathbf{a}}), \quad N_{4}(\overline{\mathbf{a}})=N_{4}(\mathbf{a}),
$$

правый и левый обратный элементы (они, конечно, существуют не всегда) равны

$$
\mathbf{a}_{\mathrm{r}}^{-1}=\frac{\overline{\mathbf{a}} N_{2}^{*}(\mathbf{a})}{N_{4}(\mathbf{a})}=\frac{\overline{\mathbf{a}} \cdot \mathbf{a}^{*} \overline{\mathbf{a}}^{*}}{N_{4}(\mathbf{a})}, \quad \mathbf{a}_{1}^{-1}=\frac{N_{2}^{*}(\overline{\mathbf{a}}) \overline{\mathbf{a}}}{N_{4}(\mathbf{a})} \equiv \mathbf{a}_{\mathrm{r}}^{-1} .
$$

Поскольку $N_{2}(\overline{\mathbf{a}})=N_{2}(\mathbf{a})$, правый и левый обратные элементы совпадают. Таким образом, обратный элемент существует для каждого элемента из алгебры $B$ с ненулевой 4-нормой.

В работе [12] 4-скалярное произведение для алгебр бикватернионов и биоктав было построено на основе формулы Шафера (25), которая для случая $n=4$ имеет вид

$$
\begin{aligned}
& (\mathbf{a}, \mathbf{b}, \mathbf{c}, \mathbf{d})=\frac{1}{24}\left[N_{4}(\mathbf{a}+\mathbf{b}+\mathbf{c}+\mathbf{d})-N_{4}(\mathbf{a}+\mathbf{b}+\mathbf{c})-N_{4}(\mathbf{a}+\mathbf{b}+\mathbf{d})-\right. \\
& \quad-N_{4}(\mathbf{a}+\mathbf{c}+\mathbf{d})-N_{4}(\mathbf{b}+\mathbf{c}+\mathbf{d})+N_{4}(\mathbf{a}+\mathbf{b})+N_{4}(\mathbf{a}+\mathbf{c})+N_{4}(\mathbf{b}+\mathbf{c})+ \\
& \left.\quad+N_{4}(\mathbf{a}+\mathbf{d})+N_{4}(\mathbf{b}+\mathbf{d})+N_{4}(\mathbf{c}+\mathbf{d})-N_{4}(\mathbf{a})-N_{4}(\mathbf{b})-N_{4}(\mathbf{c})-N_{4}(\mathbf{d})\right] .
\end{aligned}
$$

Принимая во внимание формулу (26), выражающую для алгебр бикватернионов и биоктав 4-норму через 2-норму, и выражая 2-норму суммы через 2-скалярное произведение согласно (17), получаем после ряда упрощений формулу 4-скалярного 
произведения в алгебрах с центральным сопряжением с (псевдо)нормой четвертой степени:

$$
\begin{aligned}
(\mathbf{a}, \mathbf{b}, \mathbf{c}, \mathbf{d})= & \frac{1}{6}\left[(\mathbf{a}, \mathbf{b})\left(\mathbf{c}^{*}, \mathbf{d}^{*}\right)+(\mathbf{a}, \mathbf{c})\left(\mathbf{b}^{*}, \mathbf{d}^{*}\right)+(\mathbf{a}, \mathbf{d})\left(\mathbf{b}^{*}, \mathbf{c}^{*}\right)+\right. \\
& \left.+(\mathbf{c}, \mathbf{d})\left(\mathbf{a}^{*}, \mathbf{b}^{*}\right)+(\mathbf{b}, \mathbf{d})\left(\mathbf{a}^{*}, \mathbf{c}^{*}\right)+(\mathbf{b}, \mathbf{c})\left(\mathbf{a}^{*}, \mathbf{d}^{*}\right)\right]
\end{aligned}
$$

2.6. Основные конструкции в матричном представлении. Для приложения введенных выше конструкций к геометрии и физике целесообразно представить их в матричном виде (см. также [13]). Бикватернионы имеют точное матричное представление с помощью комплексных $(2 \times 2)$-матриц. Это представление известно как алгебра матриц Паули:

$$
\begin{aligned}
& \mathbf{1}=\left(\begin{array}{ll}
1 & 0 \\
0 & 1
\end{array}\right), \quad \mathbf{q}_{1}=\left(\begin{array}{cc}
0 & -i \\
-i & 0
\end{array}\right), \quad \mathbf{q}_{2}=\left(\begin{array}{cc}
0 & -1 \\
1 & 0
\end{array}\right), \quad \mathbf{q}_{3}=\left(\begin{array}{cc}
-i & 0 \\
0 & i
\end{array}\right), \\
& \mathbf{i}_{0}=\left(\begin{array}{cc}
i & 0 \\
0 & i
\end{array}\right), \quad \mathbf{i}_{1}=\left(\begin{array}{cc}
0 & 1 \\
1 & 0
\end{array}\right), \quad \mathbf{i}_{2}=\left(\begin{array}{cc}
0 & -i \\
i & 0
\end{array}\right), \quad \mathbf{i}_{3}=\left(\begin{array}{cc}
1 & 0 \\
0 & -1
\end{array}\right) \text {. }
\end{aligned}
$$

В данном представлении бикватернион

$$
\mathbf{a}=a_{0} \cdot \mathbf{1}+a_{1} \mathbf{q}_{1}+a_{2} \mathbf{q}_{2}+a_{3} \mathbf{q}_{3}+k_{0} \mathbf{i}_{0}+k_{1} \mathbf{i}_{1}+k_{2} \mathbf{i}_{2}+k_{3} \mathbf{i}_{3}
$$

записывается так:

$$
\mathbf{A}=\left(\begin{array}{ll}
a_{11} & a_{12} \\
a_{21} & a_{22}
\end{array}\right)=\left(\begin{array}{cc}
a_{0}+k_{3}-i a_{3}+i k_{0} & -i a_{1}-a_{2}+k_{1}-i k_{2} \\
-i a_{1}+a_{2}+k_{1}+i k_{2} & a_{0}-k_{3}+i a_{3}+i k_{0}
\end{array}\right) .
$$

Основному бикватернионному сопряжению (11)

$$
\overline{\mathbf{a}}=a_{0} \cdot \mathbf{1}-a_{1} \mathbf{q}_{1}-a_{2} \mathbf{q}_{2}-a_{3} \mathbf{q}_{3}+k_{0} \mathbf{i}_{0}-k_{1} \mathbf{i}_{1}-k_{2} \mathbf{i}_{2}-k_{3} \mathbf{i}_{3}
$$

соответствует присоединенная матрица

$$
\mathbf{A}^{\prime}=\left(\begin{array}{cc}
a_{22} & -a_{12} \\
-a_{21} & a_{11}
\end{array}\right)=\left(\begin{array}{cc}
a_{0}-k_{3}+i a_{3}+i k_{0} & i a_{1}+a_{2}-k_{1}+i k_{2} \\
i a_{1}-a_{2}-k_{1}-i k_{2} & a_{0}+k_{3}-i a_{3}+i k_{0}
\end{array}\right) .
$$

2-Норма бикватерниона, понимаемая как комплексное число, равна детерминанту его матрицы, а с другой стороны, она может быть выражена через произведение матрицы бикватерниона на присоединенную матрицу $\left(\mathbf{A} \cdot \mathbf{A}^{\prime}=\operatorname{Det} \mathbf{A} \cdot I\right)$ :

$$
N_{2}(\mathbf{a})=\operatorname{Det} \mathbf{A}=\frac{1}{2} \operatorname{Sp}\left(\mathbf{A} \cdot \mathbf{A}^{\prime}\right)
$$

Отсюда 2-скалярное произведение (комплексное число) есть

$$
(\mathbf{a}, \mathbf{b})=\frac{1}{4} \operatorname{Sp}\left(\mathbf{A} \cdot \mathbf{B}^{\prime}+\mathbf{B} \cdot \mathbf{A}^{\prime}\right) .
$$

Правое 2-векторное произведение (элемент алгебры бикватернионов) имеет вид

$$
\langle\mathbf{a}, \mathbf{b}\rangle=\frac{1}{2}\left(\mathbf{A} \cdot \mathbf{B}^{\prime}-\mathbf{B} \cdot \mathbf{A}^{\prime}\right)
$$


Дуальному кватернионному сопряжению (вторая запись в (14)) соответствует эрмитово-сопряженная матрица

$$
\mathbf{A}^{+}=\left(\begin{array}{cc}
a_{11}^{*} & a_{21}^{*} \\
a_{12}^{*} & a_{22}^{*}
\end{array}\right)=\left(\begin{array}{cc}
a_{0}+k_{3}+i a_{3}-i k_{0} & i a_{1}+a_{2}+k_{1}-i k_{2} \\
i a_{1}-a_{2}+k_{1}+i k_{2} & a_{0}-k_{3}-i a_{3}-i k_{0}
\end{array}\right) .
$$

Внешнему для кватернионов комплексному сопряжению (15) (равному комбинации сопряжений а̃)

$$
\overline{\tilde{\mathbf{a}}}=\mathbf{a}^{\star}=a_{0}+a_{1} \mathbf{q}_{1}+a_{2} \mathbf{q}_{2}+a_{3} \mathbf{q}_{3}-k_{0} \mathbf{i}_{0}-k_{1} \mathbf{i}_{1}-k_{2} \mathbf{i}_{2}-k_{3} \mathbf{i}_{3}
$$

соответствует матрица

$$
\mathbf{A}^{\prime+}=\mathbf{A}^{\star}=\left(\begin{array}{cc}
a_{22}^{*} & -a_{21}^{*} \\
-a_{12}^{*} & a_{11}^{*}
\end{array}\right)=\left(\begin{array}{cc}
a_{0}-k_{3}-i a_{3}-i k_{0} & -i a_{1}-a_{2}-k_{1}+i k_{2} \\
-i a_{1}+a_{2}-k_{1}-i k_{2} & a_{0}+k_{3}+i a_{3}-i k_{0}
\end{array}\right) .
$$

Как следствие, 4-норма равна

$$
N_{4}(\mathbf{a})=\frac{1}{4} \operatorname{Sp}\left(\mathbf{A} \mathbf{A}^{\prime}\right)\left[\operatorname{Sp}\left(\mathbf{A} \mathbf{A}^{\prime}\right)\right]^{*}=\frac{1}{4} \operatorname{Sp}\left(\mathbf{A} \mathbf{A}^{\prime}\right) \operatorname{Sp}\left(\mathbf{A}^{\star} \mathbf{A}^{\star \prime}\right),
$$

а 4-скалярное произведение есть

$$
\begin{aligned}
& (\mathbf{a}, \mathbf{b}, \mathbf{c}, \mathbf{d})=\frac{1}{96}\left[\operatorname{Sp}\left(\mathbf{A} \mathbf{B}^{\prime}+\mathbf{B} \mathbf{A}^{\prime}\right) \operatorname{Sp}\left(\mathbf{C}^{\star} \mathbf{D}^{\star \prime}+\mathbf{D}^{\star} \mathbf{C}^{\star \prime}\right)+\right. \\
& \quad+\operatorname{Sp}\left(\mathbf{A} \mathbf{C}^{\prime}+\mathbf{C A}^{\prime}\right) \operatorname{Sp}\left(\mathbf{B}^{\star} \mathbf{D}^{\star \prime}+\mathbf{D}^{\star} \mathbf{B}^{\star \prime}\right)+\operatorname{Sp}\left(\mathbf{A D}^{\prime}+\mathbf{D} \mathbf{A}^{\prime}\right) \operatorname{Sp}\left(\mathbf{B}^{\star} \mathbf{C}^{\star \prime} \mathbf{C}^{\star} \mathbf{B}^{\star \prime}\right)+ \\
& \quad+\operatorname{Sp}\left(\mathbf{C D}^{\prime}+\mathbf{D C}^{\prime}\right) \operatorname{Sp}\left(\mathbf{A}^{\star} \mathbf{B}^{\star \prime}+\mathbf{B}^{\star} \mathbf{A}^{\star \prime}\right)+\operatorname{Sp}\left(\mathbf{B D}^{\prime}+\mathbf{D B}^{\prime}\right) \operatorname{Sp}\left(\mathbf{A}^{\star} \mathbf{C}^{\star \prime}+\mathbf{C}^{\star} \mathbf{A}^{\star \prime}\right)+ \\
& \left.\quad+\operatorname{Sp}\left(\mathbf{B} \mathbf{C}^{\prime}+\mathbf{C B}^{\prime}\right) \operatorname{Sp}\left(\mathbf{A}^{\star} \mathbf{D}^{\star \prime}+\mathbf{D}^{\star} \mathbf{A}^{\star \prime}\right)\right] .
\end{aligned}
$$

Все формулы значительно упрощаются в важном для приложений случае чисто мнимых бикватернионов с шестью ненулевыми компонентами $\left(a_{0}=k_{0}=0\right)$. В этом случае

$$
\overline{\mathbf{a}}=-\mathbf{a}, \quad \mathbf{a}^{\star}=-\tilde{\mathbf{a}}, \quad \text { т. е. } \quad \mathbf{A}^{\prime}=-\mathbf{A}, \quad \mathbf{A}^{\star}=-\mathbf{A}^{+} .
$$

Тогда 2-норма, 2-скалярное и 2-векторное произведения суть

$$
\begin{aligned}
N_{2}(\mathbf{a}) & =-\mathbf{a}^{2}=-\frac{1}{2} \operatorname{Sp}\left(\mathbf{A}^{2}\right), \\
(\mathbf{a}, \mathbf{b}) & =-\frac{1}{4} \operatorname{Sp}(\mathbf{A B}+\mathbf{B A}), \\
\langle\mathbf{a}, \mathbf{b}\rangle & =\frac{1}{2}(\mathbf{B A}-\mathbf{A B})=\frac{1}{2}[\mathbf{B}, \mathbf{A}], \\
\langle\mathbf{a}, \mathbf{b}\rangle^{2} & =-N_{2}(\langle\mathbf{a}, \mathbf{b}\rangle)=\frac{1}{8} \operatorname{Sp}\left([\mathbf{A}, \mathbf{B}]^{2}\right),
\end{aligned}
$$

$[\mathbf{A}, \mathbf{B}]$ - коммутатор матриц А, В. В данном случае 4-норма и 4-скалярное произведение равны

$$
N_{4}(\mathbf{a})=\frac{1}{4} \operatorname{Sp}\left(\mathbf{A}^{2}\right) \operatorname{Sp}\left(\mathbf{A}^{+2}\right)
$$




$$
\begin{aligned}
(\mathbf{a}, \mathbf{b}, \mathbf{c}, \mathbf{d})=\frac{1}{96}\left[\operatorname{Sp}(\mathbf{A B}+\mathbf{B A}) \operatorname{Sp}\left(\mathbf{C}^{+} \mathbf{D}^{+}+\mathbf{D}^{+} \mathbf{C}^{+}\right)+\right. \\
\quad+\operatorname{Sp}(\mathbf{A C}+\mathbf{C A}) \operatorname{Sp}\left(\mathbf{B}^{+} \mathbf{D}^{+}+\mathbf{D}^{+} \mathbf{B}^{+}\right)+\operatorname{Sp}(\mathbf{A D}+\mathbf{D A}) \operatorname{Sp}\left(\mathbf{B}^{+} \mathbf{C}^{+}+\mathbf{C}^{+} \mathbf{B}^{+}\right)+ \\
\quad+\operatorname{Sp}(\mathbf{C D}+\mathbf{D C}) \operatorname{Sp}\left(\mathbf{A}^{+} \mathbf{B}^{+}+\mathbf{B}^{+} \mathbf{A}^{+}\right)+\operatorname{Sp}(\mathbf{B D}+\mathbf{D B}) \operatorname{Sp}\left(\mathbf{A}^{+} \mathbf{C}^{+}+\mathbf{C}^{+} \mathbf{A}^{+}\right)+ \\
\left.\quad+\operatorname{Sp}(\mathbf{B C}+\mathbf{C B}) \operatorname{Sp}\left(\mathbf{A}^{+} \mathbf{D}^{+}+\mathbf{D}^{+} \mathbf{A}^{+}\right)\right] .
\end{aligned}
$$

\section{3. НЕКОТОРЫЕ ВОЗМОЖНОСТИ ПРИМЕНЕНИЯ ПОЛИНОРМ В ФИЗИКЕ}

Как хорошо известно [1], электромагнитное поле довольно естественно описывается бикватернионными переменными, в этом контексте логично искать первые приложения норм четвертого порядка и иных конструкций для алгебр с центральным сопряжением [13].

3.1. 4-Форма и элемент массы электромагнитного поля. Введем бикватернион электромагнитного поля

$$
\mathbf{F}=\mathbf{q}_{1} H_{1}+\mathbf{q}_{2} H_{2}+\mathbf{q}_{3} H_{3}+\mathbf{i}_{1} E_{1}+\mathbf{i}_{2} E_{2}+\mathbf{i}_{3} E_{3}=\mathbf{H}+\mathbf{i}_{0} \mathbf{E},
$$

где $\mathbf{H}$ и $\mathbf{E}$ - чисто мнимые кватернионы, которые могут рассматриваться просто как 3-векторы напряженности электрического и магнитного полей. Комплексная 2-норма этого бикватерниона в силу формулы (13) равна

$$
\begin{aligned}
N_{2}(\mathbf{F}) & =H_{1}^{2}+H_{2}^{2}+H_{3}^{2}-E_{1}^{2}-E_{2}^{2}-E_{3}^{2}+2 \mathbf{i}_{0}\left(H_{1} E_{1}+H_{2} E_{2}+H_{3} E_{3}\right)= \\
& =\mathbf{H}^{2}-\mathbf{E}^{2}+2 \mathbf{i}_{0}(\mathbf{H}, \mathbf{E})=\mathbf{I}_{\mathbf{s}}+2 \mathbf{i}_{0} \mathbf{I}_{\mathrm{p}},
\end{aligned}
$$

т. е. она составлена из скалярного $\mathbf{I}_{\mathrm{s}}=\mathbf{H}^{2}-\mathbf{E}^{2}$ и псевдоскалярного $\mathbf{I}_{\mathrm{p}}=(\mathbf{H}, \mathbf{E})$ инвариантов электромагнитного поля. В силу (27), (28) 4-норма бикватерниона электромагнитного поля (вещественный скаляр) равна

$$
\begin{aligned}
N_{4}(\mathbf{F}) & =\left(H_{1}^{2}+H_{2}^{2}+H_{3}^{2}-E_{1}^{2}-E_{2}^{2}-E_{3}^{2}\right)^{2}+4\left(H_{1} E_{1}+H_{2} E_{2}+H_{3} E_{3}\right)^{2}= \\
& =\left(\mathbf{H}^{2}-\mathbf{E}^{2}\right)^{2}+4(\mathbf{H}, \mathbf{E})^{2}=\mathbf{I}_{\mathrm{s}}^{2}+4 \mathbf{I}_{\mathrm{p}}^{2} .
\end{aligned}
$$

Чтобы выявить скрытый смысл этого выражения, используем (32):

$$
N_{4}(\mathbf{F})=\left(\mathbf{H}^{2}-\mathbf{E}^{2}\right)^{2}+4(\mathbf{E}, \mathbf{H})^{2}=\left(\mathbf{H}^{2}+\mathbf{E}^{2}\right)^{2}+4\langle\mathbf{H}, \mathbf{E}\rangle^{2}
$$

или

$$
N_{4}(\mathbf{F})=\left(\mathbf{H}^{2}+\mathbf{E}^{2}\right)^{2}-4\left|\langle\mathbf{H}, \mathbf{E}\rangle^{2}\right|
$$

(последний член меньше или равен нулю). Таким образом, 4-норма электромагнитного поля имеет ясный физический смысл: она является 2-нормой 4-вектора потока энергии-импульса поля, т. е. по сути представляет собой квадрат плотности массы поля. (Эта плотность не равна нулю в силу неаддитивности массы в релятивистской физике.) Несложно также показать, что 4-норма электромагнитного поля равна свертке тензора энергии-импульса с самим собой, $T_{i k} T^{i k}$; кроме этого, квадрату 4-нормы равен детерминант матрицы тензора энергии-импульса. 


\subsection{4-Форма и преобразование дуальности электромагнитного поля.}

Из выражения (54) видно, что 4-норма электромагнитного поля содержит скрытую симметрию кирального типа $U(1)$. Она инвариантна при замене

$$
\mathbf{E}^{\prime}=\mathbf{E} \cos \theta+\mathbf{H} \sin \theta, \quad \mathbf{H}^{\prime}=-\mathbf{E} \sin \theta+\mathbf{H} \cos \theta
$$

или, в бикватернионном виде (51),

$$
\mathbf{F}^{\prime}=\left(\mathbf{H}+\mathbf{i}_{0} \mathbf{E}\right)^{\prime}=\mathbf{F} e^{\mathbf{i}_{0} \theta} .
$$

Это обобщенное преобразование дуальности, оставляющее, как известно, инвариантными тензор энергии-импульса электромагнитного поля и уравнения Максвелла в вакууме [17]. При наличии зарядов его надо дополнить преобразованием

$$
e^{\prime}=e \cos \theta+g \sin \theta, \quad g^{\prime}=-e \sin \theta+g \cos \theta,
$$

где $g$ - магнитный заряд (частицы в этом случае несут одновременно оба типа зарядов). Таким образом, введение в рассмотрение 4-нормы электромагнитного поля позволяет прояснить природу обобщенной дуальной инвариантности уравнений Максвелла, скрытую при стандартном квадратичном подходе.

3.3. Связь 4-формы с электродинамикой Борна-Инфельда. Формула (53) для 4-нормы электромагнитного поля содержит в качестве одного из своих членов выражение, совпадающее с лагранжианом Максвелла. Что получится, если использовать 4-норму при конструировании лагранжиана поля? Оказывается, это естественным путем ведет к нелинейной электродинамике Борна-Инфельда. В самом деле, принцип соответствия подсказывает следующий вид модифицированного лагранжиана в первом нетривиальном приближении:

$$
L^{\prime}=L_{0}+\Lambda,
$$

где $L_{0}$ - лагранжиан Максвелла, а $\Lambda$ - малая в обычных условиях поправка. Таким образом, модифицированный лагранжиан, использующий 4-норму поля, должен иметь в первом постмаксвелловском приближении следующий вид:

$$
L^{\prime}=-\frac{1}{8 \pi}\left[\left(\mathbf{H}^{2}-\mathbf{E}^{2}\right)+\alpha\left(\left(\mathbf{H}^{2}-\mathbf{E}^{2}\right)^{2}+4(\mathbf{H}, \mathbf{E})^{2}\right)\right],
$$

где $\alpha$ - малый параметр. Именно такое приближение дает электродинамика БорнаИнфельда. Действительно, лагранжиан здесь, как известно, имеет вид

$$
L=-\frac{1}{4 \pi a^{2}}\left[\sqrt{1+a^{2}\left(\mathbf{H}^{2}-\mathbf{E}^{2}\right)-a^{4}(\mathbf{H}, \mathbf{E})^{2}}-1\right] .
$$

Используя разложение корня $\sqrt{1+x} \approx 1+x / 2-x^{2} / 8$ и сохраняя члены до $a^{4}$, получаем следующее соответствие с электродинамикой Максвелла:

$$
\begin{aligned}
L & \approx-\frac{1}{4 \pi a^{2}}\left(1+\frac{1}{2} a^{2}\left(\mathbf{H}^{2}-\mathbf{E}^{2}\right)-\frac{1}{2} a^{4}(\mathbf{H}, \mathbf{E})^{2}-\frac{1}{8} a^{4}\left(\mathbf{H}^{2}-\mathbf{E}^{2}\right)^{2}-1\right)= \\
& =-\frac{1}{8 \pi}\left(\left(\mathbf{H}^{2}-\mathbf{E}^{2}\right)-\frac{1}{4} a^{2}\left(\left(\mathbf{H}^{2}-\mathbf{E}^{2}\right)^{2}+4(\mathbf{H}, \mathbf{E})^{2}\right)\right),
\end{aligned}
$$

т.е. в точности представление (58). 
Преобразовав лагранжиан Борна-Инфельда к виду

$$
\begin{aligned}
L & =-\frac{1}{4 \pi a^{2}}\left[\sqrt{\left(1+\frac{a^{2}}{2}\left(\mathbf{H}^{2}-\mathbf{E}^{2}\right)\right)^{2}-\frac{a^{4}}{4}\left(\left(\mathbf{H}^{2}-\mathbf{E}^{2}\right)^{2}+4(\mathbf{H}, \mathbf{E})^{2}\right)}-1\right]= \\
& =-\frac{1}{4 \pi a^{2}}\left[\sqrt{\left.\left(1+\frac{a^{2}}{2} L_{0}\right)^{2}-\frac{a^{4}}{4} N_{4}(\mathbf{F})-1\right],}\right.
\end{aligned}
$$

можно увидеть, что сходная ситуация будет сохраняться во всех порядках: будут комбинироваться вещественная часть 2-нормы (лагранжиан Максвелла) и 4-норма электромагнитного поля (53), т. е. скаляры второго и четвертого порядков. Заметим, что требование составлять лагранжиан из скаляров второго и четвертого порядков вместе с принципом соответствия однозначно приводит к теории Борна-Инфельда лишь в первом нетривиальном порядке. В следующих порядках этих условий недостаточно, чтобы зафиксировать коэффициенты, т. е. возникает некоторый теоретический произвол (диапазон теорий). Это принципиальная ограниченность предложенного подхода. Тем не менее несомненно, что введение в рассмотрение 4-нормы электромагнитного поля выявляет естественность нелинейного обобщения электродинамики в теории Борна-Инфельда.

3.4. О лагранжиане модели Скирма. Еще один случай, когда алгебраические соображения могут прояснить полученные интуитивным путем физические результаты, дает модель Скирма [18], [19] ядерных взаимодействий. Она была создана для описания барионов как протяженных локализованных структур с нетривиальным топологическим зарядом, который интерпретируется как барионное число. Модель Скирма оказалась простым и удачным прообразом эффективной мезонной теории (в общем виде до сих пор неизвестной), к которой должна сводиться квантовая хромодинамика в низкоэнергетическом пределе. В этом пределе сильные взаимодействия в значительной мере сводятся к обмену $\pi$-мезонами. В рамках модели Скирма удается удовлетворительным образом описывать спектроскопию основных состояний адронов и их взаимодействия.

Основной объект модели - скирмионы - представляют собой киральные топологические солитоны со спонтанно нарушенной киральной симметрией. Описывающее их киральное поле $U(x)$ принимает значения на полевом многообразии модели $\Phi=S^{3} \simeq[S U(2)]$ и параметризуется изовекторным полем $\varphi^{a}(x)$, соответствующим триплету пионных полей:

$$
\begin{gathered}
U(x)=\varphi^{0}+i(\boldsymbol{\tau} \boldsymbol{\varphi})=e^{i\left(n^{a} \tau^{a}\right) \Theta(x)}, \\
n^{a}=\frac{\varphi^{a}}{|\boldsymbol{\varphi}|}, \quad \sin \Theta= \pm|\varphi|, \quad a=1,2,3,
\end{gathered}
$$

где $\boldsymbol{n}$ - единичный вектор, $\boldsymbol{\tau}$ - матрицы Паули, $\Theta(x)$ - киральный угол, $x=\left(x^{0}, \boldsymbol{x}\right)$. Поля (60) подчинены естественным граничным условиям на пространственной бесконечности:

$$
U(x) \rightarrow \boldsymbol{I} \quad\left(\varphi^{a}(x) \rightarrow 0\right) \quad \text { при } \quad|\boldsymbol{x}| \rightarrow \infty,
$$

где $\boldsymbol{I}$ - единичная $(2 \times 2)$-матрица. 
Левоинвариантные киральные токи $l_{\mu}=U^{-1} \partial_{\mu} U$ принимают значения в алгебре Ли группы $S U(2)$. Динамика модели Скирма определяется плотностью лагранжиана

$$
\mathfrak{L}=-\frac{1}{4 \lambda^{2}} \operatorname{Sp}\left(l_{\mu} l^{\mu}\right)+\frac{\varepsilon^{2}}{16} \operatorname{Sp}\left(\left[l_{\mu}, l_{\nu}\right]\left[l^{\mu}, l^{\nu}\right]\right),
$$

где $\varepsilon, \lambda$ - параметры модели. Первый член совпадает с лагранжианом Вайнберга-Гюрши, который в “древесном" приближении хорошо воспроизводит результаты алгебры токов для низкоэнергетической динамики пионов. Второй член четвертого порядка отвечает идее Скирма рассматривать барионы как вихри в пионной жидкости, описываемой "обобщенными скоростями” $l_{\mu}$.

Однако, поскольку лагранжиан модели Скирма не выводится напрямую из КХД и не является единственным обобщением лагранжиана Вайнберга-Гюрши, возникает вопрос о возможности его обоснования с помощью алгебраических соображений.

Как легко видеть, с алгебраической точки зрения каждая из компонент токов $l_{\mu}$ представляет собой чисто мнимый кватернион. Это означает, что мы имеем дело с тензорным произведением $B \otimes H$ алгебры бикватернионов $B$ (эрмитовы компоненты которой представляются матрицами Паули и отвечают пространству-времени) на алгебру кватернионов $H$. Такая алгебра не является алгеброй с центральным сопряжением, поэтому здесь приходится расширять технику, изложенную выше. Однако по-прежнему все формулы естественным образом выражаются на языке алгебры бикватернионов [13]. Можно показать, что в общем случае алгебра $B \otimes H$ обладает комплексной формой четвертой степени и вещественной формой восьмой степени. Однако в данном случае ситуация сильно упрощается благодаря тому, что ненулевыми являются только двенадцать компонент этой алгебры (по три чисто мнимых кватерниона на каждую из четырех эрмитовых компонент пространственно-временного бикватерниона). Благодаря этому псевдонорма сводится к вещественной форме четвертой степени. Опустив промежуточные вычисления, приведем конечный результат: в кватернионной записи 4-форма равна

$$
\begin{aligned}
N_{4}= & \left(\left|\boldsymbol{l}_{0}\right|^{2}-\left|\boldsymbol{l}_{1}\right|^{2}-\left|\boldsymbol{l}_{2}\right|^{2}-\left|\boldsymbol{l}_{3}\right|^{2}\right)^{2}+ \\
& +4\left(\left\langle\boldsymbol{l}_{2}, \boldsymbol{l}_{3}\right\rangle^{2}+\left\langle\boldsymbol{l}_{3}, \boldsymbol{l}_{1}\right\rangle^{2}+\left\langle\boldsymbol{l}_{1}, \boldsymbol{l}_{2}\right\rangle^{2}-\left\langle\boldsymbol{l}_{0}, \boldsymbol{l}_{1}\right\rangle^{2}-\left\langle\boldsymbol{l}_{0}, \boldsymbol{l}_{2}\right\rangle^{2}-\left\langle\boldsymbol{l}_{0}, \boldsymbol{l}_{3}\right\rangle^{2}\right) .
\end{aligned}
$$

Используя формулы (47), (48), можно перевести эту 4-форму на обычный тензорный язык:

$$
N_{4}=\frac{1}{4}\left(\operatorname{Sp}\left(l_{\mu} l^{\mu}\right)\right)^{2}+\frac{1}{2}\left(\operatorname{Sp}\left(\left[l_{\mu}, l_{\nu}\right]\left[l^{\mu}, l^{\nu}\right]\right)\right)
$$

В согласии с принципом соответствия и по аналогии с электродинамикой БорнаИнфельда естественно считать, что лагранжиан модели (в первом постлинейном приближении) должен представлять собой сумму вещественной части псевдонормы второго порядка $N_{2}=\operatorname{Sp}\left(l_{\mu} l^{\mu}\right) / 2$ и псевдонормы четвертого порядка в качестве малой поправки. Таким образом, лагранжиан в этом приближении должен иметь вид

$$
\mathfrak{L}=N_{2}+\alpha N_{4},
$$


где $\alpha$ - малый параметр, или

$$
\mathfrak{L}=-\frac{1}{4 \lambda^{2}} \operatorname{Sp}\left(l_{\mu} l^{\mu}\right)+\frac{\varepsilon^{2}}{32}\left(\left(\operatorname{Sp}\left(l_{\mu} l^{\mu}\right)\right)^{2}+2 \operatorname{Sp}\left(\left[l_{\mu}, l_{\nu}\right]\left[l^{\mu}, l^{\nu}\right]\right)\right) .
$$

Это выражение очень похоже на лагранжиан Скирма, поскольку естественным образом появляется введенный им нелинейный вихревой член. Однако в лагранжиане Скирма нет среднего члена. Поскольку это слагаемое представляет собой квадрат 2-нормы времениподобного вектора, т. е. дважды положительно, его добавление, по-видимому, может улучшить свойства модели. Подобный член уже вводился в эффективной мезонной теории (см. например, [20]), правда, с другими коэффициентами.

Как и в электродинамике Борна-Инфельда, алгебраические соображения вместе с принципом соответствия определяют вид модифицированного лагранжиана Скирма (65) лишь в первом постлинейном порядке. В общем случае лагранжиан может представлять собой некоторую функцию, комбинирующую псевдонормы второго и четвертого порядков, т.е. снова возникает диапазон теорий. В связи с этим приведем согласованный с алгебраическими соображениями вид модифицированного лагранжиана в форме, сходной с электродинамикой Борна-Инфельда:

$$
\begin{aligned}
\mathfrak{L} & =-\frac{1}{\lambda^{2}}\left[\sqrt{\left.\left(1+\frac{1}{2} N_{2}\right)^{2}-\frac{\varepsilon^{2} \lambda^{2}}{4} N_{4}-1\right]=}\right. \\
& =-\frac{1}{\lambda^{2}}\left[\sqrt{\left(1+\frac{1}{4} \operatorname{Sp}\left(l_{\mu} l^{\mu}\right)\right)^{2}-\frac{\varepsilon^{2} \lambda^{2}}{16}\left(\left(\operatorname{Sp}\left(l_{\mu} l^{\mu}\right)\right)^{2}+2 \operatorname{Sp}\left(\left[l_{\mu}, l_{\nu}\right]\left[l^{\mu}, l^{\nu}\right]\right)\right)}-1\right] .
\end{aligned}
$$

\section{4. ВЫВОДЫ}

Поскольку 4-норма для алгебр бикватернионов и биоктав однозначно выражается через 2-норму (которая также является мультипликативной), неясно, насколько существенными будут те новые возможности, которые создает переход от комплексной 2-нормы к вещественной 4-норме, а также введение 4-скалярного произведения. Тем не менее, как минимум, выявляются некоторые новые связи между уже известными фактами, которые затушеваны при стандартном квадратичном подходе.

В любом случае представляются интересными попытки придать квадраобъектам какой-либо геометрический и физический смысл. Некоторые первые шаги в этом направлении представлены здесь (прежде всего, для теории поля). При этом на ряд привычных вопросов можно посмотреть под новым углом зрения. В частности, рассмотрение связанной с бикватернионами вещественной 4-нормы показывает естественность перехода от электродинамики Максвелла к нелинейной электродинамике Борна-Инфельда. Алгебраический подход демонстрирует также естественность добавления предложенного Скирмом нелинейного члена в лагранжиан, описывающий ядерные взаимодействия посредством обмена пионами. При этом выявляется целесообразность введения дополнительного члена в лагранжиан Скирма, что предположительно может улучшить свойства модели. Таким образом, алгебраическое 
рассмотрение позволяет прояснить интуитивные находки в нелинейных полевых теориях, а также внести в них некоторые дополнительные элементы жесткости.

Благодарности. Авторы выражают свою признательность Ю.П. Рыбакову, И. В. Воловичу, участникам семинара Отдела математической физики МИРАН за ценное обсуждение. Работа частично поддержана РФФИ (грант № 68-01-00798).

\section{Список литературы}

[1] А. В. Березин, Ю.А. Курочкин, Е.А. Толкачев, Кватернионы в релятивистской физике, УРCC, М., 2003.

[2] Я. Лыхмус, Э. Паал, Л. Соргесепп, Труды института физики АН Эстонии, 66 (1990), $8-22$.

[3] W. K. Clifford, Proc. London Math. Soc., 4 (1873), 381-395.

[4] А.П. Котельников, Винтовое счисление и некоторые приложения его $к$ геометрии и механике, Типо-Литография Императорского Унив., Казань, 1895.

[5] E. Study, Geometry der Dynamen, Teubner, Leipzig, 1903.

[6] Ф. М. Диментберг, Винтовое исчисление и его приложения в механике, Наука, М., 1965.

[7] Э. Садбери, Гиперкомплексные числа в геометрии и физике, 1:2 (2004), 130-157.

[8] И. Л. Кантор, А. С. Солодовников, Гиперкомплексные числа, Наука, М., 1973.

[9] Б. А. Розенфельд, М. П. Замаховский, Геометрия групn Ли, МЦНМО, М., 2003.

[10] О.В.Мельников, В.Н. Ремесленников, В. А. Романьков, Л. А. Скорняков, И. П. Шестаков, Общая алгебра, Т. 1, Справочная математическая библиотека, ред. Л. А. Скорняков, Наука, М., 1990.

[11] Д. С. Баэз, Гиперкомплексные числа в геометрии и физике, 3:1(5) (2006), 120-176; arXiv: math/0105155.

[12] А. А. Элиович, Гиперкомплексные числа в геометрии и физике, 1:2(2) (2004), 24-50.

[13] А. А. Элиович, Гиперкомплексные числа в геометрии и физике, 5:2(10) (2008), 131-159.

[14] Р. Д. Шафер, "О формах степени $n$, допускающих композицию", Гиперкомплексные числа в геометрии и физике, 1:1 (2004), 140-154.

[15] R. D. Schafer, Adv. Math., 4:2 (1970), 127-148.

[16] K. McCrimmon, Trans. Amer. Math. Soc., 127:3 (1967), 527-551.

[17] В. И. Стражев, Л. М. Томильчик, Электродинамика с магнитным зарядом, Наука и техника, Минск, 1975.

[18] В. Г. Маханьков, Ю. П. Рыбаков, В. И. Санюк, УФН, 162:2 (1992), 1-61.

[19] Ю. П. Рыбаков, В. И. Санюк, Многомерные солитоны, Изд-во РУДН, М., 2001.

[20] В. А. Андрианов, В. Ю. Новожилов, "Скалярные мезоны в модели Скирма и солитоны с барионным зарядом", Вопросы квантовой теории поля и статистической физики, 8, Зап. научн. сем. ПОМИ, 169, Наука, Ленинградское отделение, СПб., 1988, $3-11$.

Поступила в редакцию 6.02.2009, после доработки 17.07.2009 